\title{
Application of hollow fiber centrifugal ultrafiltrate purification as the pretreatment technology for traditional Chinese medicine: its application for analysis of honokiol and magnolol in TCM preparations containing Cortex Magnoliae Officinalis
}

\author{
Jing $\mathrm{An}^{1}$, Chaoxing $\mathrm{He}^{2}$, Caihui Guo ${ }^{1}$, Zhanjun Dong ${ }^{1}$ \\ ${ }^{1}$ Department of Pharmacy, Hebei General Hospital, Shijiazhuang, China; ${ }^{2}$ Department of Pharmaceutics, School of Pharmacy, Hebei Medical \\ University, Shijiazhuang, China \\ Contributions: (I) Conception and design: J An; (II) Administrative support: Z Dong; (III) Provision of study materials or patients: Z Dong; (IV) \\ Collection and assembly of data: J An, C He; (V) Data analysis and interpretation: C Guo, J An; (VI) Manuscript writing: All authors; (VII) Final \\ approval of manuscript: All authors. \\ Correspondence to: Zhanjun Dong. Department of Pharmacy, Hebei General Hospital, 348 West Heping Road, Shijiazhuang 050051, China. \\ Email: dzjhbgh@126.com.
}

\begin{abstract}
Backgrounda Traditional Chinese medicine (TCM) preparations are very complex mixtures, and the content of bioactive components is usually very low. Therefore, before final analysis, the preparation of an appropriate sample is necessary. Sample preparation is the most time-consuming and error-prone part of the analytical procedure, and the choice of purification technology greatly influences the reliability of the final analysis.
\end{abstract}

Methods: In the present study, we evaluated the feasibility of hollow fiber centrifugal ultrafiltrate (HFCFUF) as a purification technology for the analysis of bioactive components in TCM preparations. The HFCF-UF technology was applied to analyze honokiol and magnolol in TCM preparations containing Cortex Magnoliae Officinalis (Hou Po in Chinese Pinyin). A mini centrifugal device based on hollow fiber was employed to remove the macromolecule components. A single step of simple centrifugation was required before the filtrate could be directly injected into an existing high performance liquid chromatography (HPLC) system without any further clean-up step or use of special columns. This greatly simplified the pretreatment steps, and improved the accuracy of analytic methods. The separation was achieved on a Diamonsil $\mathrm{C}_{18}$ column (i.d. $5 \mu \mathrm{m}, 150 \mathrm{~mm} \times 4.6 \mathrm{~mm}$ ) with $V$ (methanol): $V$ (acetonitrile): $V(0.5 \%$ acetic acid solution) $=44: 22: 34$ as the mobile phase at a flow rate of $1.0 \mathrm{~mL} / \mathrm{min}$.

Results: It had good linear relationship between the peak areas of honokiol and magnolol and their concentrations at $6.40-205$ and $3.15-101 \mu \mathrm{g} / \mathrm{mL}(\mathrm{r}=0.9999)$, respectively. The method recovery was over $92.6 \%$ with a relative standard deviation (RSD) of less than $3.0 \%$. The average recovery of honokiol was $97.7 \%$ with an RSD of $3.0 \%$, and that of magnolol was $96.8 \%$ with RSD of $2.8 \%$.

Conclusions: The application of HFCF-UF in TCM preparations could assist in making the quality control of TCM simple, rapid, and accurate. The HFCF-UF purification procedure can be used as an alternative means for analyzing bioactive components in TCM preparations.

Keywords: Liquid chromatography; hollow fiber centrifugal ultrafiltrate (HFCF-UF); purification technology; magnolol; honokiol; traditional Chinese medicine (TCM)

Submitted Jan 12, 2021. Accepted for publication Mar 02, 2021.

doi: 10.21037/apm-21-242

View this article at: http://dx.doi.org/10.21037/apm-21-242 


\section{Introduction}

Currently, Chinese medicines (CMs) are gaining increased attention globally due to their distinguished therapeutic effects, and the safety and efficacy of CMs have become major concerns for health authorities and the public $(1,2)$. Quality assessment and control is important for ensuring the safety and efficacy of CMs. The active components and their synergistic effects are generally understood to be responsible for the pharmacological and/or toxicological properties of CMs (3), hence, the characterization of active components is crucial for validating its clinical efficacy.

Various detection techniques have been applied in CM analysis, such as high-performance liquid chromatography (HPLC) (4), liquid chromatography-mass spectrometry (LC-MS) $(5,6)$, capillary electrophoresis (CE) (7), and gas chromatography (GC)/GC-MS $(8,9)$. However, pretreatment of the sample is indispensable prior to modern instrumental analysis, due to the complexity of the matrixes of CMs (3).

Protein precipitation, liquid-liquid extraction (LLE), and solid phase extraction (SPE) are widely applied pretreatment techniques, often necessitating complex timeconsuming multi-step procedures, including extraction, purification, drying, concentration, and so on. It has been reported that pretreatment steps greatly influence the reliability and accuracy of analysis results (10), and complex multi-step procedures can foster contamination and result in low accuracy. Besides, the primary procedure of sample pretreatment is extraction of target analytes. Generally, the extraction is a phase change process, thus mass transfer of the target analyte may be incomplete, which can easily lead to the loss of analytes and a lower recovery rate.

Moreover, sample preparation is the main source of errors and discrepancies, and preparation can greatly affect analytical precision. The variance of the result increases as the number of steps increases (11). From a variety of detailed protocols $(12,13)$, many more than 10 processing steps are required until final analysis. Therefore, reducing the number of operating steps and improving analytical precision is an urgent requirement for optimizing sample preparation.

Thirdly, the length of the entire analytical procedure is determined by sample preparation, which accounts for approximately $2 / 3$ of total analysis time (10). Productivity would be greatly increased by reductions in sample preparation time sample preparation time. The most effective way to approach this is to handle samples with as few as possible processing steps. Our team has always been committed to the development of a simpler and faster method for pretreatment of samples with complex matrixes.

The hollow fiber centrifugal ultrafiltrate (HFCF-UF) product (our patented product, composed of a tube and a u-shaped hollow fiber; project no: 2009100744294) has been used to separate the macro-molecules from complicated matrixes such as plasma $(14,15)$. The HFCF-UF is good for class separation by different molecular weight cut-off, and HPLC enables high-efficiency fractionation; therefore, HFCF-UF-HPLC has been shown to be an effective means in analysis of various components in complex matrixes.

In this study, the HFCF-UF device was employed to analyze honokiol and magnolol in Huoxiang Zhengqi Oral Liquid (HXZQOL). WE explored the feasibility of HFCF-UF technology for the determining the contents of bioactive components in traditional Chinese medicine (TCM) preparations.

The liquid herbal formula, HXZQOL, is very popular in China. It is used to induce diaphoresis and treat sunstroke. It is made up by many kinds of TCM ingredients, such as Pogostemonis Rbizoma, Cortex Magnoliae Officinalis (Chinese Pinyin: Hou Po), Pericarpium Citri Reticulatae, Poria, Rbizoma Pinelliae, and so on. Hou Po is the key ingredient of the formula. It has been reported that Hou Po has antiinflammatory, analgesic, and central-depressant effects. As the main active components of Hou Po, honokiol and magnolol are the index of quality control.

In this study, we evaluated the development and application of HFCF-UF as a purification procedure of honokiol and magnolol in TCM preparations before LC analysis. In contrast with traditional methods, this method is simple, rapid, and low cost. After just 1 step of centrifugation by our device, the filtrate could be directly injected onto the HPLC system for analysis. Thus, it greatly simplified the pretreatment steps, and improved the sensitivity of analytic methods.

We present the following article in accordance with the MDAR checklist (available at http://dx.doi.org/10.21037/ apm-21-242).

\section{Methods}

\section{Instrumentation}

The HPLC system consisted of a system controller (CTO10A), pump (LC-10AT.vp), UV detector (SPD-10A), and electronic integrator (Class-vp) (all from Shimazu, Kyoto, 


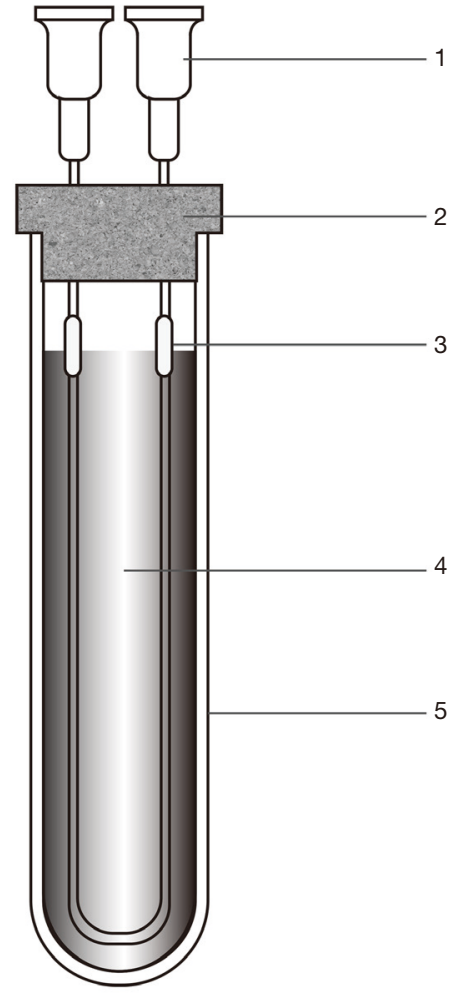

Figure 1 Hollow fiber centrifugal ultrafiltration device. 1, syringe needle; 2, rubber bung; 3, hollow fiber; 4, sample solution; 5, centrifuge tube.

Japan). The analytical column used was a Diamonsil $\mathrm{C}_{18}$ (Dikma Tech., Foothill Ranch, CA, USA) $(150 \mathrm{~mm} \times$ $4.6 \mathrm{~mm}$ ID, $5 \mu \mathrm{m}$ particles).

\section{Reagents and materials}

The standard of honokiol and magnolol were purchased from the National Institute for Food and Drug Control (Beijing, China). We obtained HXZQOL from Tong Ren Tang Technologies Co., Ltd. (Beijing, China). The HPLC-grade methanol and acetonitrile were acquired from Fisher Scientific (Fair Lawn, NJ, USA). Ultrapure water was obtained from a Milli-Q water purification system (Millipore, Bedford, MA, USA).

Polypropylene hollow fiber membrane $(200 \mu \mathrm{m}$ wall thickness, molecular cut off 6,000 Da) was from FoShan Power-full Enviromental Protection Technology Co., Ltd. (FoShan, China). Hollow fibers were cut into $15 \mathrm{~cm}$ segments, sonicated in methanol then ultrapure water, and dried before use.

\section{Chromatographic conditions}

Separation of honokiol and magnolol was achieved on the Diamonsil $\mathrm{C}_{18}$ column with the detector set at $294 \mathrm{~nm}$ and the column maintained at $30{ }^{\circ} \mathrm{C}$. The mobile phase was a mixture of $44: 22: 34 \mathrm{v} / \mathrm{v} / \mathrm{v}$ methanol-acetonitrile- $0.5 \%$ acetic acid solution, and the flow rate was maintained at $1 \mathrm{~mL} / \mathrm{min}$. The sample injection volume was $10 \mu \mathrm{L}$.

\section{Standard solutions}

A combined stock solution of honokiol $(820 \mu \mathrm{g} / \mathrm{mL})$ and magnolol $(404 \mu \mathrm{g} / \mathrm{mL})$ was prepared in methanol and stored at $4{ }^{\circ} \mathrm{C}$. A second dilution was made to prepare a combined standard solution containing honokiol $205 \mu \mathrm{g} / \mathrm{mL}$ and magnolol $101 \mu \mathrm{g} / \mathrm{mL}$. Working dilutions in methanol were prepared when needed.

\section{Sample preparations}

We diluted $1 \mathrm{~mL}$ HXZQOL to $10 \mathrm{~mL}$ with methanol. About $8 \mathrm{~mL}$ of the obtained solution was placed in a centrifuge tube. A HFCF-UF device (Figure 1) was placed in the sample solution. Finally, the tube was centrifuged for $15 \mathrm{~min}$ at $3.8 \times 10^{3} \mathrm{~g}$. After ultrafiltration, the solution obtained in the lumen of the hollow fiber was withdrawn into the syringe and $10 \mu \mathrm{L}$ was injected in the HPLC system.

\section{Statistical analysis}

The precision of the method was evaluated by 6 determinations of the same test solutions, as RSD considering acceptability less than $5 \%$. Accuracy of the method was calculated by recovery experiments using the standard addition technique. The recoveries were calculated by $\left(\mathrm{A}_{\text {found }}-\mathrm{A}_{\text {added }}\right) / \mathrm{A}_{\text {added }} \times 100 \%$ (A is analyts).

\section{Results}

\section{HPLC chromatograms}

Under the conditions described above, the HPLC chromatograms of standard solution with honokiol at concentration of $205 \mu \mathrm{g} / \mathrm{mL}$ and magnolol at $101 \mu \mathrm{g} / \mathrm{mL}$, and the test sample solution are shown in Figure 2. The retention time for honokiol and magnolol were approximately 13.9 and $22.8 \mathrm{~min}$, respectively. The peaks were sharp and symmetrical with good baseline resolution 

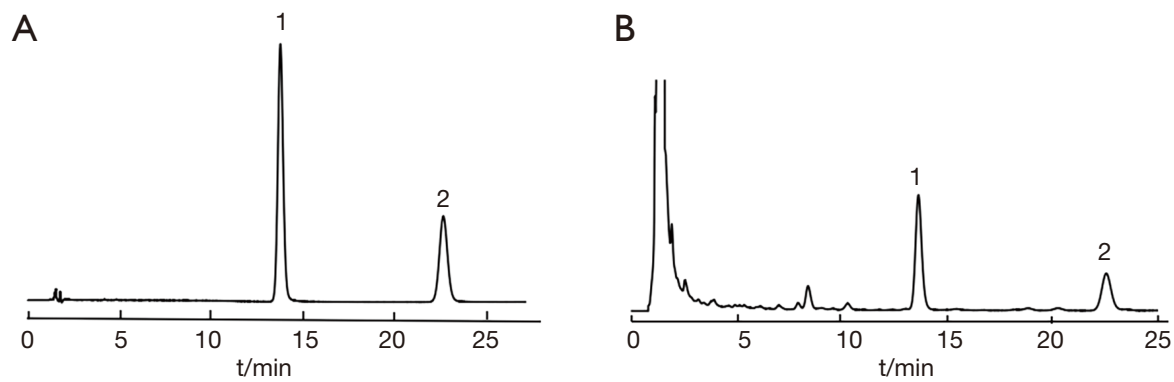

Figure 2 HPLC chromatograms of mixed reference substances (A) and sample treated by HFC-UF (B); 1, honokiol; 2, magnolol. HPLC, high performance liquid chromatography; HFC-UF, hollow fiber centrifugal ultrafiltrate.

and minimal tailings, thus facilitating accurate measurement of the peak areas. No interfering peaks were observed at the retention time window of honokiol or magnolol.

\section{Method validations}

Linearity, repeatability, precision, recovery, detectionquantification limits, and stability were used to evaluate the performance of the proposed HFCF-UF-HPLC method.

The combined standard solution of honokiol and magnolol was diluted to give a series of standard solutions (6.40-205 $\mu \mathrm{g} / \mathrm{mL}$ for honokiol and 3.15-101 $\mu \mathrm{g} / \mathrm{mL}$ for magnolol) for the calibration curves. Typical regression equations of the calibration cures were $y=1.26 \times 10^{4} x-5.38 \times 10^{3}(r=0.9999)$ for honokiol and $y=1.25 \times 10^{4} x-1.53 \times 10^{3}(r=0.9999)$ for magnolol. The limits of detection (LOD) and limits of quantification (LOQ) were calculated as 3 and 10 times the signal-to-noise ratio, respectively. The LOD was measured to be $0.32 \mu \mathrm{g} / \mathrm{mL}$ and the LOQ was $0.16 \mu \mathrm{g} / \mathrm{mL}$ for both analytes with relative standard deviation (RSD) less than $10 \%$. These results indicated that the proposed method is applicable for determination of honokiol and magnolol in herbal samples.

The precision of the method was evaluated by 6 determinations of the same test solutions, as RSD considering acceptability less than $5 \%$, and the RSD of the honokiol and magnolol were less than $1.6 \%$ and $1.9 \%$, respectively. Repeatability was assessed by 6 determinations in 1 day. Repeatability for the 2 analytes proved to be less than $2.2 \%$ and $2.5 \%$, respectively.

Accuracy of the method was calculated by recovery experiments using the standard addition technique. The recoveries were determined by spiking the mixed working solutions of standard at the concentration level of honokiol $93.0 \mu \mathrm{g} / \mathrm{mL}$ and magnolol $44.2 \mu \mathrm{g} / \mathrm{mL}$ into HXZQOL test solutions. Relative recoveries were 92.6-101.7\%, with a variation of less than $5 \%$ for the 2 analytes. The results are shown in Table 1.

Stability of the sample solutions were tested $0,2,4,6$, 8 , and $12 \mathrm{~h}$ post preparation. Under the stated conditions, stock solutions were shown to be stable for at least $12 \mathrm{~h}$.

\section{Sample analysis}

Quantitative analysis of the 2 analytes in HXZQOL was performed by use of the developed method. A total of 3 independent series of HXZQOL samples were analyzed, and samples from each series were measured 3 times. Results are presented in Table 2.

\section{Discussion}

\section{Selection of the bollow fiber}

Different types of the hollow fiber (polypropylene, polyvinylidene difluoride) were compared in the experiments. It is widely accepted that non-specific adsorption (NSA) of analyte onto the membrane is a universal phenomenon in membrane isolation techniques, and NSA can significantly affect the outcome of the assay in sample analyses. Hollow fiber membrane is some kind of polymer material, and the compound may be physically adsorbed to the membrane by van der Waals force. It means that the hollow fiber may have NSA to the analytes. The extent of the NSA was evaluated by a specific recovery, which can be expressed by the ratio of analyte concentration in the filtrate after HFCF-UF to the initial analyte concentration in the aqueous sample. The recoveries for honokiol and magnolol obtained by polypropylene hollow fiber were $100.7 \%$ and $100.4 \%$ respectively, and were $102 \%$ and $98.3 \%$, respectively, by polyvinylidene difluoride 
Table 1 Assay results of recoveries

\begin{tabular}{|c|c|c|c|c|c|c|}
\hline Component & Original, mg/mL & Added, mg/mL & Found, $\mathrm{mg} / \mathrm{mL}$ & Recovery, \% & Average, \% & RSD, \% \\
\hline \multirow{3}{*}{ Honokiol } & 46.5 & 41.0 & 87.4 & 99.8 & & \\
\hline & 46.5 & 41.0 & 88.2 & 101.7 & & \\
\hline & 46.5 & 41.0 & 86.0 & 96.3 & & \\
\hline \multirow[t]{3}{*}{ Magnolol } & 22.1 & 20.2 & 41.5 & 95.0 & 96.8 & 2.8 \\
\hline & 22.1 & 20.2 & 42.0 & 98.5 & & \\
\hline & 22.1 & 20.2 & 42.2 & 99.5 & & \\
\hline
\end{tabular}

$\mathrm{RSD}$, relative standard deviation.

Table 2 Determination of honokiol and magnolol in Huoxiang Zhengqi Oral Liquid ( $\mathrm{n}=3$ )

\begin{tabular}{|c|c|c|c|c|c|c|c|c|}
\hline Batch No. & \multicolumn{2}{|c|}{ Honokiol (the method) } & \multicolumn{2}{|c|}{ Magnolol (the method) } & \multicolumn{2}{|c|}{ Honokiol (16) } & \multicolumn{2}{|c|}{ Magnolol (16) } \\
\hline 0144760 & 998 & 1.7 & 504 & 2.2 & 985 & 0.9 & 500 & 1.3 \\
\hline 0144776 & 930 & 1.9 & 442 & 2.0 & 919 & 1.5 & 450 & 1.8 \\
\hline
\end{tabular}

$\mathrm{RSD}$, relative standard deviation.

hollow fiber. The results demonstrated that both types of hollow fiber have no NSA to the 2 analytes. Given that the mechanical stability of the polypropylene hollow fiber was superior, the experiments were finally performed using polypropylene hollow fiber.

\section{Selection of the relative centrifugal force}

In the HFCF-UF procedure, the transmembrane pressure was provided by the relative centrifugal force (RCF), and the efficiency of ultrafiltration was correlated with the RCF. The RCF was optimized to obtain the most efficient filtration. Samples, prepared according to the section of 'Sample Preparations', were centrifuged at 700-7.8x $10^{3} \mathrm{~g}$ for $15 \mathrm{~min}$, respectively. Resultingly, in a prefixed time, too weak a force would result in a small volume of filtrate which could not fulfill the lumen of the hollow fiber, and with the increase of the RCF the volume of filtrate increased. Consequently, $3.8 \times 10^{3} \mathrm{~g}$ was used for subsequent experiments because it had already provided enough filtrate for analysis.

\section{Effect of dilution agents}

The experiment was carried out using methanol and alcohol as the dilution agents to prepare the sample solutions. A higher permeability for the honokiol and magnolol was obtained using methanol as the dilution agent than with alcohol. This was probably due to the likelihood of honokiol and magnolol combining with each other in an alcohol solution to form a supramolecular assembly and increase the apparent molecular weight over the molecular weight cut-off (MWCO) of the hollow fiber. During the ultrafiltration process, they could not pass though the membrane, thus leading to low measurement results for the target analytes. 
Another possible explanation is it might bind with some coexisting materials to form a composite and be retained by the hollow fiber, resulting in a low permeability for the honokiol and magnolol. Meanwhile, when methanol is used as a diluent solvent, it can not only remove the bulk of foreign substances which are high polarity and present a minor dissolubility in methanol or alcohol, but also can destroy the polymerization status of the analytes. As a result, the permeability of honokiol and magnolol would be increased, and the analytical results obtained would be more accurate.

\section{Comparison with the traditional extraction method}

Based on the literatures, SPE (17) and LLE $(16,18)$ have been widely used to extract the target analytes from sample solution for clean-up purpose. SPE use expensive materials, are labor-consuming, usually have carry over effects. On the other hand, in the case of LLE, the main disadvantages are the use of large amounts of potentially toxic and normally expensive organic solvents, it is time-consuming and the manipulation of evaporation to dryness can easily lead to the loss of the analytes.

In some reports (19-22), a diluted sample was directly injected into the HPLC system to measure the analytes. In the chromatography analysis of herbal extracts, stationary phase compaction is consistently challenging, as the coexisting high molecular components can accumulate on the head of the column and shorten its service life. As an analytical sample, HXZQOL is a very complex matrix. The macromolecules that may exist in HXZQOL are mainly from Poria and Rhizoma Pinelliae, in which there are lots of polysaccharides and proteins. In addition, liquid sample solutions also contain small amounts of high molecular solutes, such as gummi, enzyme, and so on. If these solutes were injected onto the HPLC system, it would impact upon the performance of the column analysis and further influence the accuracy and the precision of the analytical results. In the present study, with the new HFCFUF device, the macromolecule components were easily removed and the filtrate was directly injected onto the HPLC system for analysis without further manipulation. In addition, there was no cross-contamination during sample preparation because each segment of the hollow fiber was single use, according to the very low costs of hollow fiber. The method is simple, rapid, and environmentally friendly, and it provides a simple and cheap pretreatment means for the analysis of honokiol and magnolol in HXZQOL.

\section{Comparison with the conventional commercial available UF device}

Traditional ultrafiltration technology is mainly used in the production of TCM preparations, in which large-scale equipment is employed. In this work, a homemade small device used for the separation and purification of small molecular target compounds with small samples $(3-5 \mathrm{~mL})$, so as to be further analyzed by liquid chromatography. At present, there are some reports of commercial ultrafiltration devices for determining protein binding rate and free drug concentration in plasma, but no reports for the pretreatment of TCM samples.

The HFCF-UF method is facile, utilizing both centrifugal ultrafiltration force and a hollow fiber membrane to retain high molecular solutes. Compared to the conventional commercially available UF device, the direction of centrifugal force is parallel to the hollow fiber, so the concentration polarization on the membrane has been eliminated. Due to the type of ecto-entad filtering process, ultrafiltration performance, pore size, and MWCO might not be influenced by the centrifugal force. This stability made it suitable for pretreatment of viscous sample solutions, especially the herbal sample solutions rich with macromolecule components. It is also beneficial for improving the efficiency of the column performance and the durability of the method.

\section{Conclusions}

In this study, a simple 1-step purification method using our patent device HFCF-UF without any further clean-up steps was proposed for analyzing honokiol and magnolol in TCM preparations. The pretreatment step and time were all limited, which tangibly improved the reliability and accuracy of analysis results.

The HFCF-UF method (15) is mainly applied to the purification process of aqueous samples. However, some water-insoluble components, for some reason (maybe the interaction of the complex matrix), dissolve in sample solutions. For the analysis of such samples, LLE and SPE are usually used for purification. Here, the present study for the first time applied the HFCF-UF method in organic solution as a sample pretreatment, the proposed method was applied to analysis of honokiol and magnolol in methanol, and a good analytical performance was achieved. This provides a new, simple means for the separation and analysis of the nonpolar components in sample matrixes 
rich in macromolecule components.

\section{Acknowledgments}

Funding: None.

\section{Footnote}

Reporting Checklist: The authors have completed the MDAR checklist. Available at http://dx.doi.org/10.21037/apm-21242

Data Sharing Statement: Available at http://dx.doi. org/10.21037/apm-21-242

Conflicts of Interest: All authors have completed the ICMJE uniform disclosure form (available at http://dx.doi. org/10.21037/apm-21-242). Dr. JA reports that she has a patent 2009100744294 issued. The other authors have no conflicts of interest to declare.

Ethical Statement: The authors are accountable for all aspects of the work in ensuring that questions related to the accuracy or integrity of any part of the work are appropriately investigated and resolved. This study did not involve human or animal experimentation, and there were no ethical issues that needed to be addressed.

Open Access Statement: This is an Open Access article distributed in accordance with the Creative Commons Attribution-NonCommercial-NoDerivs 4.0 International License (CC BY-NC-ND 4.0), which permits the noncommercial replication and distribution of the article with the strict proviso that no changes or edits are made and the original work is properly cited (including links to both the formal publication through the relevant DOI and the license). See: https://creativecommons.org/licenses/by-nc-nd/4.0/.

\section{References}

1. World Health Organization. WHO Guidelines on Safety Monitoring of Herbal Medicines in Pharmacovigilance Systems, World Health Organization, Geneva, 2004.

2. World Health Organization. WHO Guidelines for Assessing Quality of Herbal Medicines with Reference to Contaminants and Residues, World Health Organization, Geneva, 2007.

3. Huang Y, Tang G, Zhang T, et al. Supercritical fluid chromatography in traditional Chinese medicine analysis. J Pharm Biomed Anal 2018;147:65-80.

4. Wu D, Chen X, Hu S, et al. Study on major antitumor components in Yinchenhao decoction in vitro and in vivo based on hollow fiber cell fishing coupled with high performance liquid chromatography. J Chromatogr B Analyt Technol Biomed Life Sci 2017;1060:118-25.

5. Wong MY, So PK, Yao ZP. Direct analysis of traditional Chinese medicines by mass spectrometry. J Chromatogr B Analyt Technol Biomed Life Sci 2016;1026:2-14.

6. Ning $Z$, Liu $Z$, Song $Z$, et al. A single marker choice strategy in simultaneous characterization and quantification of multiple components by rapid resolution liquid chromatography coupled with triple quadrupole tandem mass spectrometry (RRLC-QqQ-MS). J Pharm Biomed Anal 2016;124:174-88.

7. Zhang L, Zhang W, Chen G. Determination of arbutin and bergenin in Bergeniae Rhizoma by capillary electrophoresis with a carbon nanotube-epoxy composite electrode. J Pharm Biomed Anal 2015;115:323-9.

8. Liu Q, Kong W, Qiu F, et al. One-step extraction for gas chromatography with flame photometric detection of 18 organophosphorus pesticides in Chinese medicine health wines. J Chromatogr B Analyt Technol Biomed Life Sci 2012;885-6:90-6.

9. Kong $\mathrm{W}$, Zhang $\mathrm{X}$, Shen $\mathrm{H}$, et al. Validation of a gas chromatography-electron capture detection of T-2 and HT-2 toxins in Chinese herbal medicines and related products after immunoaffinity column clean-up and precolumn derivatization. Food Chem 2012;132:574-81.

10. Hyötyläinen T. Critical evaluation of sample pretreatment techniques. Anal Bioanal Chem 2009;394:743-58.

11. Krüger T, Lehmann T, Rhode H. Effect of quality characteristics of single sample preparation steps in the precision and coverage of proteomic studies--a review. Anal Chim Acta 2013;776:1-10.

12. Li P, Zhao P, Liu W, et al. Determination of common ginsenosides in Kang'ai injection by aqueous two-phase extraction with deep eutectic solvents and HPLC-UV/ DAD. Microchem J 2018;137:302-8.

13. Guo C, Gao YG, Zang P, et al. Simultaneous determination of sixteen ginsenosides in Panax ginseng and its preparation by HPLC. Chin Tradit Herbal Drugs 2017;45:2009-13.

14. Li JM, Jiang Y, Sun T, et al. Hollow-fiber ultrafiltration then centrifugation for LC analysis of water-soluble sucrose in a water-soluble high-molecular-mass gel matrix. Chromatographia 2009;70:1023. 
15. Li JM, Li C, Jiang Y, et al. Pretreatment of plasma samples by a novel hollow fiber centrifugal ultrafiltrate device for the determination of cefaclor concentrations in human plasma. J Chromatogr A 2010;1217:6824-8.

16. Committee of National Pharmacopoeia, Pharmacopoeia of P.R. China, 2015:1729.

17. Zhang HJ, Guo HZ, Yang XP, et al. SPE-HPLC determination of honokiol and magnonol in Huoxiangzhengqi oral solution. Chin J Pharm Anal 2009;29:316-9.

18. Xia LZ, Zhang MF, Ni JW. Determination on the contents of Magnolol and Honokiol in Huoxiang Zhengqi Tincture by QAMS (Quality Assurance Management System). Journal of Guangxi University of Chinese Medicine 2017;20:49-52.

19. Chen JF, Guo D, Dong ZL, et al. Simultaneous determination of 9 Components in Huoxiang Zhengqi

Cite this article as: An J, He C, Guo C, Dong Z. Application of hollow fiber centrifugal ultrafiltrate purification as the pretreatment technology for traditional Chinese medicine: its application for analysis of honokiol and magnolol in TCM preparations containing Cortex Magnoliae Officinalis. Ann Palliat Med 2021;10(6):6359-6366. doi: 10.21037/apm-21-242
Oral Liquid by HPLC. China Pharmacy 2019;30:2957-62.

20. Cai HK, Liu XF, Jiang FR, et al. Simultaneous determination of 6 effective components in Huoxiang Zhengqi liquid by RP-HPLC. Central South Pharmacy 2014;12:577-81.

21. Liu YL, Zhao ZX, Li DM, et al. Simultaneous determination of seven components in Huoxiang Zhengqi liquid by ultra-performance liquid chromatography. Journal of China Pharmaceutical University 2013;44:249-52.

22. Nie LX, He YQ, Yu JD, et al. Rapid quality evaluation of Huoxiang Zhengqi Tincture(II): Simultaneous determination of seven components in Huoxiang Zhengqi Tincture by UPLC with wavelength switching detection. Chin Pharm J 2017;52:2192-5.

(English Language Editor: J. Jones) 